\title{
The Human Coparental Bond Implicates Distinct Corticostriatal Pathways: Longitudinal Impact on Family Formation and Child Well-Being
}

\author{
Eyal Abraham', Gadi Gilam ${ }^{2,3}$, Yaniv Kanat-Maymon ${ }^{4}$, Yael Jacob $^{2,5}$, Orna Zagoory-Sharon', \\ Talma Hendler ${ }^{2,3,5}$ and Ruth Feldman*, 1,6 \\ 'Department of Psychology and The Gonda Brain Research Center, Bar-Ilan University, Ramat-Gan, Israel; ${ }^{2}$ Functional Brain Center, Wohl Institute \\ of Advanced Imaging, Tel Aviv Sourasky Medical Center, Tel Aviv, Israel; ${ }^{3}$ School of Psychological Sciences, Faculty of Medicine, Tel Aviv University, \\ Tel Aviv, Israel; ${ }^{4}$ Interdisciplinary Center, Herzlia, Israel; ${ }^{5}$ Sagol School of Neuroscience, Tel Aviv University, Tel Aviv, Israel; ${ }^{6}$ Child Study Center, Yale \\ University School of Medicine, New Haven, CT, USA
}

\begin{abstract}
Alloparental care, the cooperative care of offspring by group members other than the biological mother, has been widely practiced since early hominin evolution to increase infant survival and thriving. The coparental bond - a relationship of solidarity and commitment between two adults who join their effort to care for children - is a central contributor to children's well-being and sociality; yet, the neural basis of coparenting has not been studied in humans. Here, we followed 84 first-time co-parents (42 couples) across the first 6 years of family formation, including opposite-sex and same-sex couples, measured brain response to coparental stimuli, observed collaborative and undermining coparental behaviors in infancy and preschool, assayed oxytocin (OT) and vasopressin (AVP), and measured coparenting and child behavior problems at 6 years. Across family types, coparental stimuli activated the striatum, specifically the ventral striatum and caudate, striatal nodes implicated in motivational goal-directed social behavior. Psychophysiological interaction analysis indicated that both nodes were functionally coupled with the vmPFC in support of the human coparental bond and this connectivity was stronger as collaborative coparental behavior increased. Furthermore, caudate functional connectivity patterns differentiated distinct corticostriatal pathways associated with two stable coparental behavioral styles; stronger caudate-vmPFC connectivity was associated with more collaborative coparenting and was linked to OT, whereas a stronger caudate-dACC connectivity was associated with increase in undermining coparenting and was related to AVP. Finally, dyadic path-analysis model indicated that the parental caudate-vmPFC connectivity in infancy predicted lower child externalizing symptoms at 6 years as mediated by collaborative coparenting in preschool. Findings indicate that the coparental bond is underpinned by striatal activations and corticostriatal connectivity similar to other human affiliative bonds; highlight specific corticostriatal pathways as defining distinct coparental orientations that underpin family life; chart brainhormone-behavior constellations for the mature, child-orientated coparental bond; and demonstrate the flexibility of this bond across family constellations and its unique contribution to child well-being.

Neuropsychopharmacology (2017) 42, 230 I-23I3; doi: I 0.1038/npp.20 I7.7I; published online 17 May 2017
\end{abstract}

\section{INTRODUCTION}

It has long been known that 'it takes a village to raise a child'. Throughout human history and across cultural communities, infants have been raised by the collaboration of several adults who joined their effort to care for children (Hrdy, 1999). Although the 'traditional' family has recently undergone profound and rapid changes (US Census Bureau, 2014) and many children are no longer reared by their biological mother and father, human children still benefit from the collaborative care of two adults (Silverstein and Auerbach,

\footnotetext{
*Correspondence: Professor R Feldman, Child Study Center, Yale University School of Medicine, New Haven, CT 065 19, USA, Tel: +203785-2540, E-mail: feldman.ruth@gmail.com

Received 16 November 2016; revised 28 March 2017; accepted 30 March 2017; accepted article preview online 12 April 2017
}

1999). These childrearing adults, whether or not the child's biological parents or a marital couple, must form a coparental bond, defined as a relationship of solidarity, coordination, and commitment to the child's well-being that is distinct from the marital relationship (Feinberg, 2003; McHale and Irase, 2011).

Human infants require the longest period of dependence for growth and maturation; hence, the male-female bond and cooperative care of the young evolved to extend much beyond copulation into a long-lasting partnership that increases infant protection and buttresses maturation of social skills (Geary and Flinn, 2001; Hrdy, 1999; Wilson, 2014). The coparental bond, therefore, is a central contributor to infant survival since the dawn of humanity. Understanding the neurobiology of coparenting may shed light on a unique bond of growing importance at a period 
when the traditional family is being substituted by multiple forms of alloparenting and the relationship between caregiving adults provides children the first exemplar of human social collaboration.

Mutually supportive and collaborative coparental relationship promotes children's well-being (Leroy et al, 2013). Children reared by collaborative coparents display less behavior problems, better emotion regulation, more advanced socialization, and less aggression as compared to those reared by non-collaborative caregivers (Teubert and Pinquart, 2010). Overall, research has described two coparental styles: collaborative coparenting is based on solidarity, coordinated action, and mutual trust which promote family cohesion, buffer stress, and foster social development (Teubert and Pinquart, 2010). In contrast, undermining coparenting is characterized by avoidance, mistrust, and competition (Caldera and Lindsey, 2006) that increase vigilance, evoke conflict, and lead to child maladjustment (McHale and Irase, 2011). Here, we aimed to elucidate the neural pathways underlying the two styles and their long-term impact on family formation and child adaptation.

Research in humans and animal models describes the key role of corticostriatal pathways in supporting mammalian affiliative bonds, including the parent-child and pair bonds (Feldman, 2017; Numan and Young, 2016). These neural pathways were found to activate in fMRI studies that expose humans to their attachment targets, including infants, romantic partners, or close friends (Abraham et al, 2014, 2016; Feldman, 2015, 2017). The striatum co-activates with several cortical structures implicated in social processing, particularly the ventromedial/medial-orbital prefrontal cortex (PFC) and dorsal anterior cingulated cortex (dACC), via complex corticostriatal networks that provide motivation for the initiation and maintenance of affiliative bonds (Calzavara et al, 2007; Haber and Knutson, 2009). Such corticostriatal pathways likely underpin the human coparental bond.

The human striatum, including the caudate, putamen, and nucleus accumbens, is a central node of the subcortical motivation/reward circuitry that supports multiple social goals by integrating reward- and aversion-related learning, motivation, motor control, and habit formation (BáezMendoza and Schultz, 2013; Delgado, 2007). The striatum contains distinct subzones in terms of functionality and connectivity with cortical regions, and these underlie discrete aspects of human sociality (Pauli et al, 2016). Although the striatum is involved in immediate reward anticipation via limbic-brainstem circuits (Haber and Knutson, 2009), it also supports higher order social behavior by providing incentive for long-term social goals (Van den Bos, 2015), and using reward outcomes to guide social cognitions, plan future actions (Delgado, 2007), and monitor the stable aspects of social life (Tops et al, 2014), functions linked with the more mature, child-focused coparental bond.

Research has identified two distinct projections from the frontal cortex to corresponding targets within the striatum with dissociable social functions (Milad and Rauch, 2012; Pauli et al, 2016; Schultz, 2000). The first connects the striatum to the ventromedial/medial-orbital PFC (hereby termed vmPFC), a cortical region activating to safety cues (Mobbs et al, 2010) and implicated in empathy, affect regulation, and the positive valence of stimuli (Vrtička et al,
2011). Striatum-vmPFC connectivity has been linked with overcoming social temptations (Rilling et al, 2002), reciprocated altruism (Rilling et al, 2004), and adaptation of behavior to that of others (Bault et al, 2011), and is reduced in anhedonia and dysphoria (Keller et al, 2013; Sabatinelli et al, 2015). Specifically, caudate-vmPFC connectivity is associated with cooperation (Rilling et al, 2002), reward regulation (Haber and Knutson, 2009), flexible goal-directed behavior, considering multiple outcomes to guide behavior (de Wit et al, 2012), and delayed gratification (Benningfield et al, 2014). The second coritcostratal pathway links the striatum with the dACC, a cortical region associated with physical and psychological pain (Eisenberger et al, 2003), social rejection (Somerville et al, 2006), fear and anxiety (Mechias et al, 2010), and experienced distress (Rainville, 2002). Striatum-dACC connectivity is related to thinking about envied others (Takahashi et al, 2009); caudate-dACC connectivity is involved in competition and conflict (Aupperle et al, 2015); and both activate when subjects punish defectors in economic games (Zink et al, 2008) or perceive a familiar person as 'foe' (Vrtička et al, 2009). A similar pattern has been found in meta-analyses of emotional states (Wager et al, 2008; Lindquist et al, 2015), with more dorsal cortical regions associated with negative emotional experiences while medial-orbital regions with positive ones.

In the current 6-year longitudinal study, we examined for the first time the neural basis of coparenting in relation to coparental behavior, social neuropeptides, and child behavior outcomes. Two-parent families (opposite- and same-sex couples) and their firstborn infant were seen four times across the first 6 years of parenthood. In the first time-point (infancy-Time1), we visited families at home, videotaped triadic whole-family interactions coded for collaborative and undermining coparental behavior, and assayed oxytocin (OT) and vasopressin (AVP). Several days later, we imaged each parent's brain response to 'partner-as-coparent' stimuli. When children were 3-4 years (preschool-Time2), we revisited families and videotaped couples in two coparenting-related dialogs, similarly coded for the two coparental styles. When children were 6 years (school-entry-Time3) parents reported on coparenting and child behavior problems.

Five hypotheses were formulated. First, we hypothesized that coparental stimuli would elicit striatal activations, similar to those found in all other human bonds. Second, we expected distinct corticostriatal pathways to be associated with the two coparental styles, such that striatal-vmPFC connectivity would be linked with collaborative coparenting and striatal-dACC with undermining parenting. Consistent with prior research (Gable et al, 1995), the two coparental styles were expected to be individually stable across childhood. Third, in light of research in humans and other mammals showing involvement of OT in trust and cooperation (Ditzen et al, 2009; Kosfeld et al, 2005) and of AVP in competition, anxiety, and suspicion (MeyerLindenberg et al, 2011), we expected that collaborative coparenting would correlate with OT while undermining coparenting with AVP. Fourth, the parent's corticostriatal connectivity that supports solidarity, cooperation, and empathy in infancy would predict lower child behavioral problems at 6 years. Finally, we expected that the path leading from the parent's coparental behavior in infancy to child behavioral problems at 6 years would be mediated by 
the parent's corticostraital circuits, affiliation hormones, and coparenting behavior at the preschool stage.

\section{MATERIALS AND METHODS}

\section{Participants}

A total of 84 first-time parents raising their infant within a partnered relationship participated (mean age time1:35.3 \pm 4.22 years (SD)): 20 couples of heterosexual biological parents (12 breast-feeding mothers) and 22 couples of homosexual fathers living within a committed two-parent family who had a child through surrogacy and were raising infants without maternal involvement since birth. All couples were living together throughout the 6-year period (mean relationship at time1: $5.38 \pm 2.419$ years). Infants (mean age at time1: $11 \pm 5.46$ months (SD); mean age at time2: $43 \pm 4.25$ months (SD); time3: $79 \pm 3.52$ months (SD)) were born at term and were healthy since birth. Parents were screened for high depression and anxiety symptoms using the beck inventory (BDI) (Beck, 1978) and the State-Trait Anxiety Inventory (STAI) (Spielberger and Lushene, 1970). Data from two fathers were excluded due to strong movement artifacts (see Supplementary section). Data from three additional parents were excluded from the current analyses because their partners did not participate in the study. All families were of middle-class background and participants completed 12-19 years of education (mean: $17.77 \pm 1.79$ years). No differences in socioeconomic status was found between same-sex and opposite-sex families. All participants, recruited through advertisement in the community, were healthy and free of medication. Participants were compensated for their time and gave written informed consent. The study was approved by the Ethics Committee of the Tel Aviv Sourasky Medical Center.

\section{Procedure}

The experimental procedure included four sessions with each family. In the first, we visited 42 families at home (Time1), salivary samples were collected for OT and AVP (2 OT and 5 AVP samples were missing for insufficient saliva), each parent was videotaped interacting with the infant separately, and families were videotaped in a triadic two-parent-andinfant interaction, coded for coparental behaviors. A few days later, each parent underwent functional brain scanning with the individually tailored home videotapes as fMRI stimuli. In the third session $($ Time $2=$ Preschool) we revisited families at home. Couples were videotaped in two 10-min coparental conversation discussing a topic of continuous childrearing conflict, and pleasant topic of mutual joy in their coparental relationship. In the fourth session (Time3 $=$ School entry), when children were 6 years parents completed self-report measures of coparenting and child behavior problems. Eight couples were lost to attrition at Time3 mainly due to inability to locate families or time constraints.

\section{Oxytocin and Vasopressin Collection and Determination}

Collection and determination of OT and AVP were conducted in accordance with our prior research
(Abraham et al, 2014, 2016; Weisman et al, 2013). For details, see Supplementary Methods.

\section{Self-Report Measures}

Two measures with good reliability and validity were used to assess the coparental bond (Time3). From the coparenting relationships scale (CRS; Feinberg et al, 2012), we used the 'Endorse Partner Parenting' and 'Coparenting Undermining' factors $(\alpha=0.90)$. From the fatherhood research and practice network coparenting relationship scale (FRPN CRS; Dyer et al, 2015) we used the 'Undermining' and 'Alliance' factors $(\alpha=0.91)$.

The child behavior checklist (CBCL) 6-18 (Achenbach, 1991 ) is the most widely used instrument identifying behavior problems in 6-18-year old children. The externalizing $(\alpha=0.89)$ and internalizing $(\alpha=0.87)$ scales were used. Children's externalizing and internalizing standard scores ranged from 33 (non-clinical range) to 59 (subclinical range) and 34 (non-clinical range) to 59 (subclinical range), respectively. None scored above the cutoff for clinical externalizing and internalizing problems $(>64)$.

\section{Coding}

Coparenting behavior (Time1 and Time2) were coded offline using the coding interactive behavior manual (CIB) (Feldman, 1998). The CIB is a well-validated global rating system for social interactions with good psychometric properties rated on Likert scale of $1=$ low to $5=$ high (Feldman, 2012). In infancy, we used the family interaction codes to code collaborative and undermining coparenting, consistent with prior research (Feldman, 2007; Feldman and Masalha, 2010; Vakart et al, 2017). In preschool, we used the adult-adult version of the CIB, which includes 33 codes applied to adult-adult discussion paradigms and has been validated in prior research using the same paradigms (Schneiderman et al, 2012, 2014; Feldman et al, 2014; Lebowitz et al, 2017). The collaborative coparenting averaged the following codes: supportive presence/communication, cooperation-mutual adaptation, acknowledgment, elaboration, gaze, expressing empathy, and positive affect. The undermining coparenting included negative effect, withdrawal-avoidance, competition, intrusiveness, and criticism. Trained raters blind to all other information coded with inter-rater reliability, measured on $20 \%$ of the sample, was intraclass $r=0.92$ (range $=0.85-0.97$ ).

\section{Functional MRI Data Acquisition and Analyses}

Imaging was performed on a GE-3 T Sigma Horizon echospeed scanner with resonant gradient echoplanar imaging system. Functional $\mathrm{T} 2{ }^{*}$-weighted images were obtained using field of view $=220 \mathrm{~mm}$, matrix size $=96 \times 96$, repetition time $=3,000 \mathrm{~ms}$, echo time $=35 \mathrm{~ms}$, flip angle $=90^{\circ}$, acquisition orientation of the fourth ventricle plane, 39 axial slices of 3-mm thickness, and gap $=0$. In addition, each functional scan was accompanied by a three-dimensional (3D) anatomical scan using anatomical 3D sequence spoiled gradient echo sequences obtained with high-resolution of $1 \times 1 \times 1 \mathrm{~mm}$. The fMRI data were analyzed with the BrainVoyager analysis package (version 2.1; Brain 
Innovation). After standard preprocessing (Supplementary Methods) statistical maps were prepared for each participant using a general linear model (GLM), in which the various blocks were defined as distinct predictors. Single-participant analysis was followed by multi-participant analysis computed with random effects using gray-matter mask. Regressors were convolved with canonical two-gamma hemodynamic response function. The baseline was considered as the averaged BOLD signal collected at all rest periods throughout the paradigm. Additional nuisance regressors included the head movement realignment parameters and the time course of averaged activity in cortical white-matter. We also incorporated a gray-matter mask and corrected for temporal autocorrelations using second-order autoregressive model. Correction of brain activation maps for multiple comparisons was performed by setting a voxel-level threshold at $P<0.001$ (uncorrected; Woo et al, 2014) and running BrainVoyager's cluster-size threshold procedure (Goebel et al, 2006). The approach implements an iterative procedure based on Monte Carlo simulation for estimating cluster-level false-positive rates. After 1000 iterations, the minimum cluster-size threshold that yielded a cluster-level falsepositive rate (alpha) of $5 \%$ in FDR, was applied to the statistical maps. Here the calculated cluster-size threshold that was applied was 10 contiguous functional voxels (where each voxel corresponds to a functional volume of $3 \mathrm{~mm}^{3}$ ).

\section{fMRI Experimental Design}

While lying in the scanner, participants were instructed to watch a series of attachment-related vignettes presented on the screen. All videos included multi-modal, dynamic, and realistic stimuli of bonding-related stimuli in the home ecology. Each parent's video set was individually tailored, comprising three 2-min infant- parent- and partner- related videos with alternating rest fixation periods of 15 or $18 \mathrm{~s}$ between stimuli, preceded by a 1-min rest with fixation period. The two clips included vignettes of: (i) each parent interacting with her/his own infant ('Self-Infant Interaction'); (ii) video clip of the partner (the second parent) interacting with his/her infant ('partner-Infant Interaction'). Order of stimuli presentation was counterbalanced into three possible sequences. To ensure that parents' and infants' affective states did not differ between parent-infant interaction vignettes (Self-Infant, Partner-Infant), we selected only clips where infants and parents were in neutral affective state (coded by CIB). To examine generalizable brain responses at the group level, analysis combined 84 different PartnerInfant interaction videos as one 'Partner-Infant Interaction' condition and 84 different parent-infant interaction videos as one 'Self-Infant Interaction' condition.

\section{Regions of Interest}

We extracted mean parameter estimates (beta values) for further analyses only for region of interest (ROIs) of a priori predictions: Caudate, ventral striatum, dACC and vmPFC. Beta values were averaged across ROI voxels and for each experimental condition separately. We used an index of 'Partner-Infant Interaction' minus 'Self-Infant Interaction' beta values to assess the mean parameter estimate specific for ROI's in response to partner 'as-a-coparent' (partner interaction with his/her own infant). ROI analyses were conducted on the brain area identified by the whole-brain GLM analysis and psychophysiological interaction (PPI) analysis (Supplementary Tables S2 and S4). We defined the ROIs using a Gaussian mask with a $4-\mathrm{mm}$ radius around the peaks of activation. Associations between ROI's activation and coparental behavioral and hormonal data were assessed using Pearson correlations and reported at $P<0.05$ (Bonferroni-corrected for multiple comparisons; 32 comparisons, $P<0.00156)$.

\section{Functional Connectivity Analysis}

Differences in functional connectivity between 'PartnerInfant Interaction' > 'Self-Infant Interaction' conditions were examined using an in-house generalized psychophysiological interaction (PPI) analysis tool previously implemented in our lab for brainvoyager (Gilam et al, 2015). A separate whole-brain random effects GLM was created for each ROI including the same nuisance regressors as detailed above. The generated maps also included as covariate the two coparental behavioral styles, the collaborative and undermining. Correction for PPI maps were set at an identical threshold as detailed for activation maps above. Pearson correlation are reported at $P<0.05$ (Bonferroni-corrected for multiple comparisons; 32 comparisons).

\section{Dyadic Analytic Strategy}

Data from couples present special analytic challenges because the lack of independence among partners (Kenny et al, 2006). To address this issue, we used IBM SPSS AMOS v.23 a structural equation modeling (SEM) software. The SEM enables to estimate path coefficients describing theoreticallybased complex network of relationships while accounting for dyadic dependencies. Moreover, SEM measures of model fit indices help assess how well a given model accounts for the relationships among partners, as well as relationships among scores of each individual. Non-significant chi-square value, CFI and TLI greater than 0.95, and RMSEA lower than 0.06 index excellent model fit (Kline, 2005).

Prior to estimating the model, we tested whether heterosexual partners were distinguishable by gender using independent $t$-tests (Supplementary Table S1) and found no gender effects, and thus treated all couples interchangeably in the SEM (Assad et al, 2007; Olsen and Kenny, 2006). Treating members as 'interchangeable' implies that assignment of participants to category of partner A or partner B is arbitrary (Kenny et al, 2006). This assignment requires imposing constrains on model paths, means, intercepts, and variances across respondent and partner, and also requires corrections for model fit indices (Olsen and Kenny, 2006). As a result of these constrains, the model path coefficients provide estimates of the intraclass covariances, the appropriate measure of association for dyadic data (Griffin and Gonzalez, 1995; Woody and Sadler, 2005).

To examine indirect association (mediation effects) between study variables we used Hayes's (2013) guidelines. Accordingly, the significance of an indirect effect is estimated using the $95 \%$ confidence interval (CI) of the cross-product of the predictor-to-mediator path and the mediator-to-outcome path. As recommended by Hayes (2013), we used 5000 
Table I Longitudinal Zero-Order Correlations Between Study Variables and Dyadic Pairwise-Correlations on the Diagonal

\begin{tabular}{|c|c|c|c|c|c|c|}
\hline & & I & 2 & 3 & 4 & 5 \\
\hline \multicolumn{7}{|c|}{ Collaborative coparental style } \\
\hline Timel (infancy) & 2. Collaborative coparenting behavior (triadic interaction) & $0.586 *(84)$ & -0.208 & & & \\
\hline Time2 (preschool) & 3. Collaborative coparenting behavior (coparental dialog) & $0.523 *(84)$ & $0.494 *(84)$ & 0.068 & & \\
\hline Time3 (school) & 4. CRS questionnaire (endorsement of partner's parenting) & $0.370 *(68)$ & $0.724 *(68)$ & $0.384 *(68)$ & 0.297 & \\
\hline \multicolumn{7}{|c|}{ Undermining coparental style } \\
\hline \multirow[t]{2}{*}{ Timel (infancy) } & I. Caudate-dACC functional connectivity & -0.094 & & & & \\
\hline & 2. Undermining coparenting behavior (triadic interaction) & $0.57 \mid *(84)$ & -0.082 & & & \\
\hline Time2 (preschool) & 3. Undermining coparenting behavior (coparental dialog) & $0.389 *(84)$ & $0.556 *(84)$ & 0.004 & & \\
\hline
\end{tabular}

$* P<0.05$ Bonferroni-corrected.

bootstrapped samples to estimate the bias-corrected and accelerated $95 \%$ CI of the indirect path. When zero is not included in the $95 \% \mathrm{CI}$, it implies a significant statistical effect at $\alpha<0.05$ (Preacher and Hayes, 2008).

\section{RESULTS}

\section{Intercorrelations Among Coparenting Measures Across the First 6 Years of Parenthood}

Pearson correlations (Table 1) assessed longitudinal associations between coparenting measures. As seen, parents showing collaborative coparenting during play in infancy (Time1), also exhibited greater collaboration during coparental discussions in preschool (Time2), and reported greater collaboration at 6 years (Time3). Similar stability emerged for undermining coparenting, suggesting that these two orientations are individually stable across time and measurement method.

\section{Whole-Brain GLM}

We conducted whole-brain analysis to identify regions showing significant brain activations across all parents stimulated specifically by observing partner-infant interaction ('Partner-Infant Interaction' condition) compared to self-infant interaction ('Self-Infant Interaction' condition). This analysis pinpoints the specific brain response to the partner in the parental role (partner as 'coparent'), while controlling for activations in response to observing ones' own infant and in response to observing social interaction. As expected, results revealed activations in the caudate and ventral striatum, key regions of the motivation-reward circuitry (Figure 1 and Supplementary Table S2). Since no differences between opposite-sex and same-sex couples were found in brain and behavior, groups were collapsed (Supplementary Table S3).

\section{Functional Connectivity}

To explore relationships between each striatal node- caudate and ventral striatum and the entire brain, we next opted for

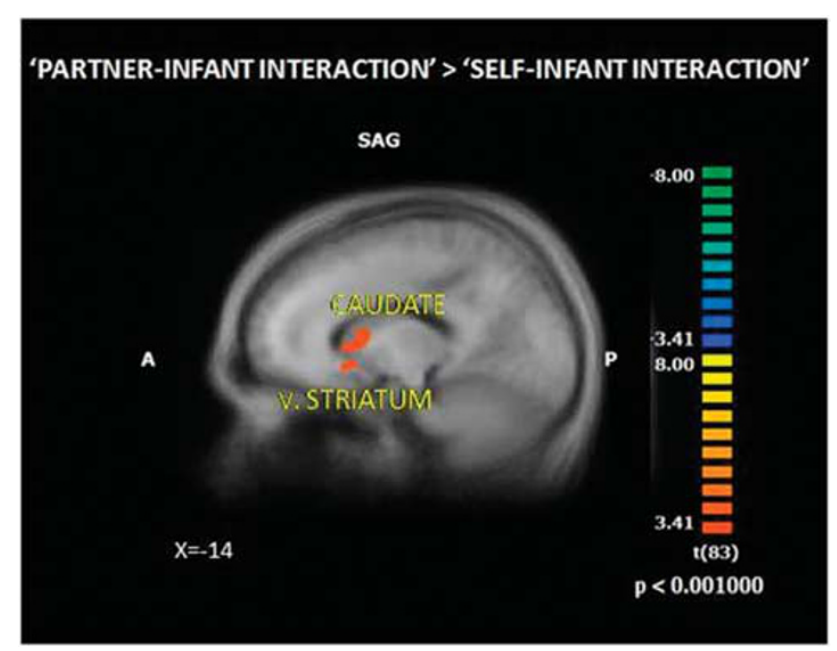

Figure I Whole-brain GLM analysis ('Partner-Infant Interaction'> 'SelfInfant Interaction') revealed activation in the caudate $(x, y, z=-16,10,12)$ and v.striatum $(x, y, z=-19,10,-3), n=84$. Brain activations are illustrated in Talairach space on an anatomical template averaging all study participants. v.straitum, ventral striatum.

condition-dependent functional connectivity analysis using PPI. We used caudate and ventral striatum as seed regions in different PPI analyses for the 'Partner-Infant Interaction $>$ Self-Infant Interaction' contrast including as covariates each coparental behavior scores (Time1, collaborative and undermining). Collaborative covariate was run separately from the undermining covariate analysis. As predicted, in the PPI analysis using caudate as a seed region, we found change in functional connectivity between caudate and vmPFC, lateral orbotofrontal cortex (lOFC), dorso-lateral PFC (dlPFC) and ventral striatum and more so as collaborative coparental scores increased (Figure 2a1 and a2; Supplementary Table S4). We also found change in functional connectivity between caudate and dACC, and more so as undermining coparental scores increased (Figure $2 \mathrm{~b} 1$ and b2; Supplementary Table S4). In the PPI analysis using ventral striatum as a seed region, we found 


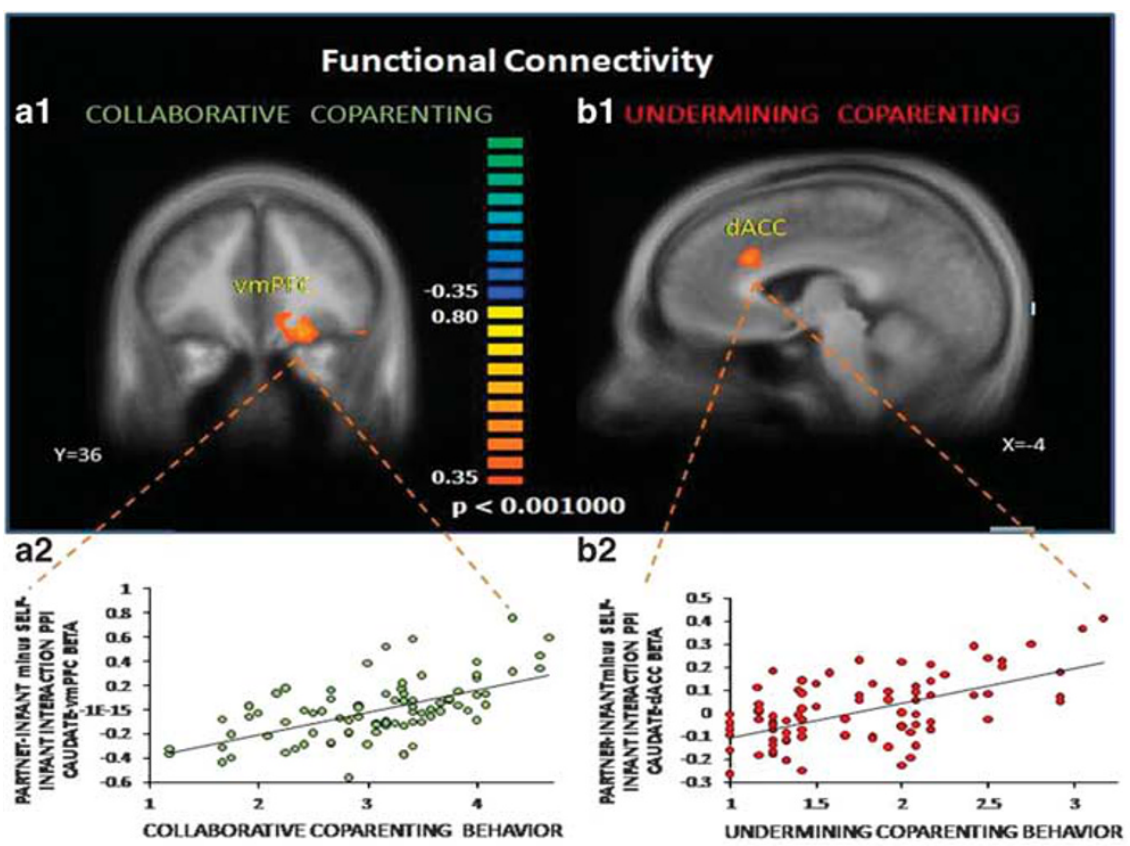

Figure 2 Functional connectivity between caudate and VmPFC and AACC and their associations with coparental behaviors. Using caudate as a seed region, differences in functional connectivity between 'Partner-Infant Interaction' minus 'Self-Infant Interaction' conditions were examined using psychophysiological interaction (PPI) while including the coparental behaviors as covariate (time I) $(n=84)$. $\left(\mathrm{a}_{1}\right)$ revealed an increase in functional connectivity between the caudate and $\operatorname{vmPFC}(x, y, z=-19,34,-9)$ as collaborative coparental behavior increased. $\left(b_{1}\right)$ revealed an increase in functional connectivity between the caudate and dACC $(x, y, z=-4,26,3 \mathrm{I})$ as undermining coparental behavior increased. $\left(a_{2}, b_{2}\right)$ Averaged $\beta$ values across spherical ROI were calculated for each subject separately for 'Partner-Infant Interaction' and 'Self-Infant Interaction' conditions, which were then subtracted ('Self-Infant Interaction' from 'PartnerInfant Interaction') for each subject. Brain activations are illustrated in Talairach space on an anatomical template averaging all study participants.

change in functional connectivity between ventral striatum and vmPFC and IOFC and more so as collaborative coparental scores increased (Supplementary Table S4), but no changes in relation to undermining coparenting. It thus appears that while both caudate and ventral striatum increased connectivity with vmPFC to coparental stimuli, only the caudate differentiated corticostriatal pathways as mediated by coparental behaviors. Thus, we continued to investigate corticostriatal pathways and their long-term correlates only with the caudate as seed region (for more PPI analyses see Supplementary Results).

\section{Longitudinal Brain-Hormones-Behavior Associations}

Distinct brain-hormone-behavior constellations over time were assessed with correlational analysis. As depicted in Table 1, for all parents, degree of caudate-vmPFC functional connectivity during the 'Partner-Infant Interaction' minus 'Self-Infant Interaction' conditions (Time1) correlated with collaborative coparental behavior at Time1 and Time2 and with positive perception of coparenting at Time3. Conversely, degree of caudate-dACC connectivity during the 'Partner-Infant Interaction' minus 'Self-Infant Interaction' condition (Time1) correlated with undermining coparental behavior at Time1 and Time2 and with negative perception of the coparental relationship at Time3. As to hormones, OT correlated with caudate-vmPFC connectivity $(r=0.520$, $P<0.05$ Bonferroni-corrected; Figure 3a), but not with caudate-dACC $(r=-0.015, P>0.5)$, whereas AVP was associated with caudate-dACC connectivity $(r=0.445$, $P<0.05$ Bonferroni-corrected; Figure $3 \mathrm{~b}$ ), but not with
caudate-vmPFC connectivity $(r=-0.129, P>0.2)$. Furthermore, collaborative coparenting correlated with OT ( $r=0.303, \quad P<0.05$ Bonferroni-corrected), but not with AVP $(r=-0.019, P>0.8)$, and undermining coparenting correlated with AVP $(r=0.265, P<0.05$ uncorrected $)$, but not with OT ( $r=-0.021, P>0.8)$ (Supplementary results).

\section{Associations Between Parent Functional Connectivity and Child Behavioral Problems at 6 Years}

A significant negative correlation was found between parent's caudate-vmPFC connectivity and child's externalizing problems $(r=-0.396, P<0.05$ Bonferroni-corrected; Figure 3c), but not with internalizing behavior $(r=0.052$, $P>0.5)$. No significant correlations were found between caudate-dACC connectivity and child's behavioral problems (Externalizing: $r=0.009, P>0.5$; Internalizing: $r=-0.161$, $P>0.1)$ (see Supplementary section).

\section{Dyadic Path Model}

For a comprehensive longitudinal model combining brain, hormones, and behavior, we constructed a dyadic path model (Figure 4) leading from coparental behaviors in infancy to behavioral problems at 6 years, as mediated by brain connectivity, affiliation hormones, and coparental behavior in preschool controlling for the dyadic level. The theoretical model fitted the data adequately, $\chi^{2}(150)=126.21$, $P=0.921, \mathrm{CFI}=1.00, \mathrm{TLI}=1.06$, RMSEA $=0.00$. As seen, undermining coparenting at Timel was associated with AVP, which, in turn, was linked to caudate-dACC 


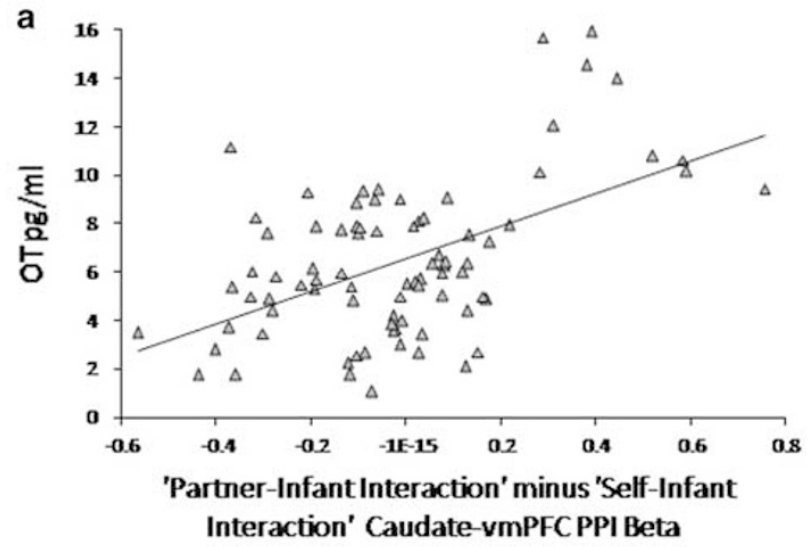

b

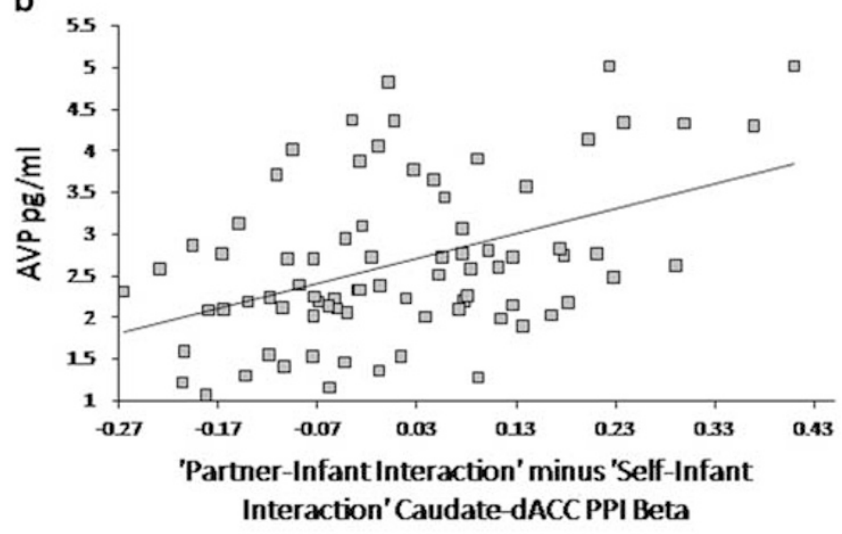

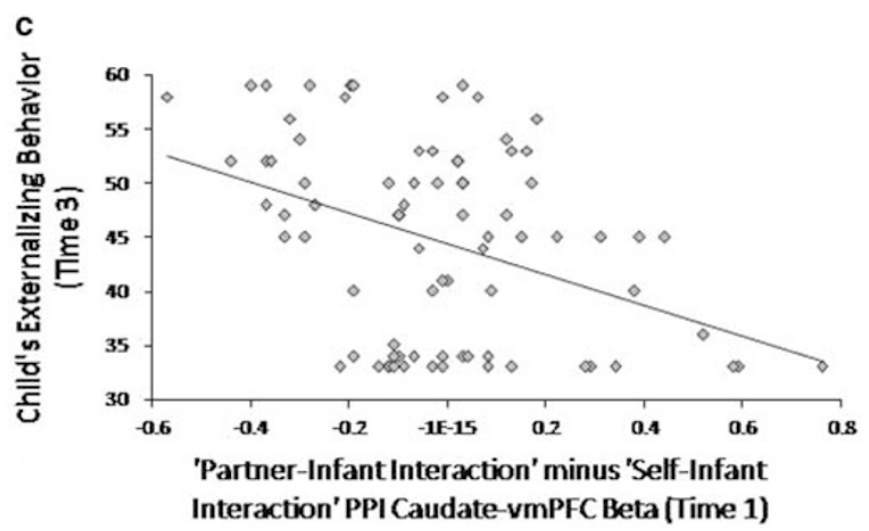

Figure 3 (a) Scatter plots show significant correlations between caudate-vmPFC functional connectivity, during 'Partner-Infant Interaction' minus 'Self-Infant Interaction' condition and OT levels $(r=0.520, P<0.05$ Bonferroni-corrected, $n=82)$. (b) Scatter plots show significant correlations between caudate-dACC functional connectivity during 'Partner-Infant Interaction' minus 'Self-Infant Interaction' condition and AVP levels $(r=0.445, P<0.05$ Bonferroni-corrected, $n=79$ ). (c) Scatter plots show that greater caudate-vmPFC functional connectivity during 'Partner-Infant Interaction' minus 'Self-Infant Interaction' conditions

(Time I = infancy) predicts lower child's externalizing behavior (time $3=$ school-entry) $(r=-0.396, P<0.05$ Bonferroni-corrected, $n=68)$.

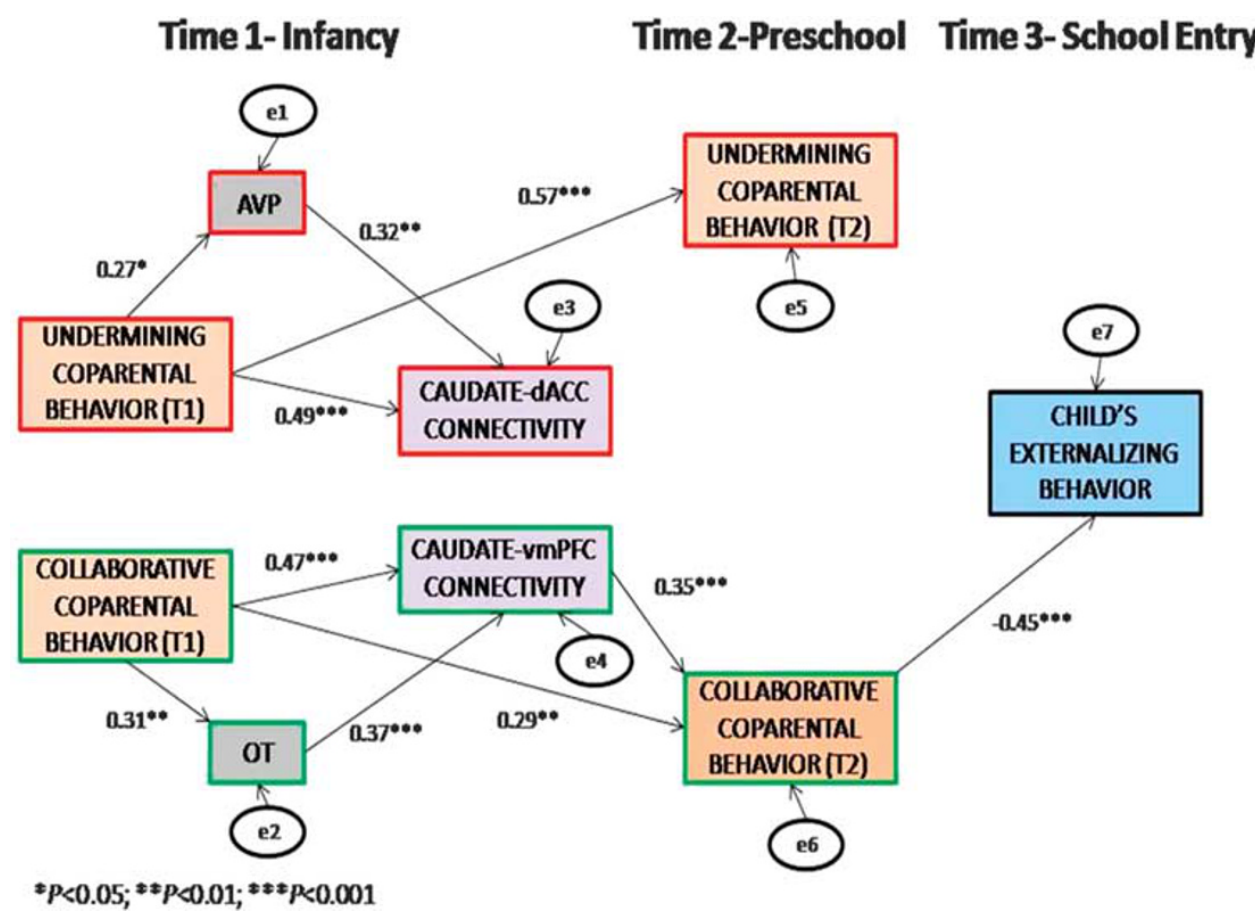

Figure 4 Dyadic path model leading from coparental behaviors to child's behavioral problems as mediated by corticostriatal circuits, affiliation hormones, and coparental behaviors in preschool. 
connectivity. The significance of the indirect path from undermining coparenting at Time1 to caudate-dACC connectivity through AVP was examined using 5000 bootstrapped samples to estimate the bias-corrected and accelerated $95 \%$ CI of this indirect path. The fact that the 95\% CI did not contain zero (indirect $\beta=0.086, P<0.05$, $95 \% \mathrm{CI}=0.001,0.222$ ) and that undermining coparenting at Time1 was also directly associated with caudate-dACC connectivity indicates that AVP partially mediated the link between undermining coparenting in infancy and caudatedACC connectivity. In addition, undermining coparenting at Time1 predicted undermining coparenting at Time2.

Collaborative coparenting at Time1 was associated with OT, which, in turn, was linked with caudate-vmPFC connectivity. The $95 \%$ CI of the indirect effect indicated that OT partially mediated the link between collaborative coparenting at Timel and caudate-vmPFC connectivity (indirect $\beta=0.115, P<0.05,95 \% \mathrm{CI}=0.001,0.169$ ). Collaborative coparenting at Time1 was directly linked with caudate-vmPFC connectivity and collaborative coparenting at Time2. Moreover, caudate-vmPFC connectivity was associated with collaborative coparenting at Time 2 and this, in turn, was linked with child externalizing behavior at Time3. Test of indirect effect suggested that coparental behavior at Time2 mediated the link between caudatevmPFC connectivity and child externalizing behavior (indirect $\beta=-0.158, P<0.05,95 \% \mathrm{CI}=-0.271,-0.044$ ).

\section{DISCUSSION}

As inter-birth intervals grew shorter, infant dependence longer, and female reproductive cycle increased from that of the Great Apes, hominin mothers needed a system of alloparental care to assure the survival and growth of codependent infants of increasingly larger brains (Gettler, 2014). As both allomothering and father presence improved infant survival and reduced infant abandonment, it is argued that unless mothers were able to trust and cooperate with other group members, including male partners, to provide care for their slow-maturing young, the human species could not have evolved (Hrdy, 2011). Yet, despite its centrality, this is the first study to assess the neurobiological basis of the human coparental bond, which has its roots in the ancient cooperative breeding system. We followed first-time coparent couples across the first 6 years of parenthood to chart neural networks, affiliation hormones, and observed coparental behaviors in the natural habitat in opposite-sex and same-sex couples raising their firstborn child within a twoparent family and tested their long-term associations with child behavior problems. Before discussing the results, it is important to note that our findings, although longitudinal, are correlational in nature and do not suggest causality, and the term 'effects' describes statistical, not causal effects.

Our findings indicate that the neural underpinnings of the coparental bond resemble those of other human bonds, suggesting commonalities across social bonds in activating the 'affiliative brain', which has its roots in the mammalian maternal network (Feldman, 2015, 2016; Numan and Young, 2016). Imaging studies of human attachments, whether parental, romantic, or close friendships, which exposed individuals to stimuli of their loved ones, indicate that attachment bonds are underpinned by striatal activity and these activations of dopamine-related pathways provide subcortical motivation for the formation of affiliative bonds. In humans, however, such evolutionarily ancient striatal activity is connected via multiple ascending and descending projections to specific cortical sites pending on the specific bond and shaped through ongoing bonding-related behavior (For review, Feldman, 2017). It is thus possible that across evolution, the mammalian maternal network has been repurposed to support numerous affiliative bonds that enable life in social groups, and that the long-term cooperative breeding and alloparental relationships were based on similar neural circuits, fine-tuned to support the mature, child-focused coparental bond.

In search for specific corticostriatal pathways that define the integration of subcortical nuclei with cortical structures and coparenting behaviors, our findings highlight six important aspects of the human coparental bond. First, the coparental bond is indeed underpinned by two important nodes of the subcortical dopaminergic motivation/reward circuit, the caudate and ventral striatum. Second, wholebrain PPI analysis showed that both the caudate and ventral striatum are functionally coupled with a fronto-medial circuit comprising the vmPFC, dlPFC, and lOFC, describing links between striatal subzones and cortical structures implicated in reciprocity, safety, and empathy. Such corticostriatal connectivity increased as coparental behaviors were marked by greater collaboration, adaptation, and mutual respect. Third, only the caudate showed distinct functional connectivity patterns with cortical sites that were sensitive to specific coparental behavior; caudate coupling with vmPFC was stronger with the increase in collaborative coparenting, whereas caudate connectivity with dACC increased as the undermining style became more salient. Fourth, two prototypical brain-hormone-behavior constellations emerged; the first linked the caudate with vmPFC, correlated with OT, and expressed behaviorally as collaborative coparenting, the second involved caudate coupling with dACC, correlated with AVP, and was related to undermining coparenting. Importantly, those coparental styles were individually stable across both time and measurement method. Fifth, connectivity patterns had long-term implications for children's adaptation and the parent's caudatevmPFC connectivity predicted lower children's externalizing problems at 6 years. Finally, our dyadic path model described how brain, hormones, and behavior coalesce across the first years of parenthood to shape stable patterns of family relationships and modes of child adaptation.

It has long been suggested that organisms utilize subtle cues in their environment to alter the pathways of adaptive development and such cues become active agents in shaping offspring phenotype and facilitating evolutionary transitions (Gilbert et al, 2015). We suggest that subtle differences in the interactions between caregivers and the brain circuits and endocrine systems associated with them are the first salient stable cues infants observe in their social ecology. Since the neural balance in some families tilts toward collaboration, empathy, and cooperation and in others toward competition, vigilance, and avoidance, continuous exposure to these patterns may exert cumulative impact on adaptation. Coparenting is central predictor of children's adaptation from infancy to adolescence (Teubert and Pinquart, 2010), 
with supportive coparenting promoting security, social skills, and emotion regulation (Altenburger et al, 2015; Farr and Patterson, 2013), whereas uncoordinated coparenting increasing emotional difficulties and externalizing problems (LeRoy et al, 2013; McHale and Rasmussen, 1998; Teubert and Pinquart, 2010). It has been theorized that children's social development is shaped by two classes of mechanisms; 'participation' in the parent-child relationship, and 'observation' of the relationship between parents (Feldman et al, 2010; Schneider et al, 1989). Our findings add the neural underpinnings of the 'observation' mechanism, that is, the neural correlates of coparental interactions to which children are exposed, and chart their implications across the first years of life.

The striatum-a hub serving as both input zone for basal ganglia and integration port from multiple cortical networks-is critical for motivational goal-directed behavior, action planning, evaluating others' behavior, weighing 'approach-reward' $v s$ 'avoidance-threat' responses, and decision making in social contexts (Grahn et al, 2008; O'Doherty et al, 2004; Pauli et al, 2016; Robinson et al, 2012). The caudate is implicated in long-term romantic love for opposite- and same-sex partners (Acevedo et al, 2011; Zeki and Romaya, 2010), parental behavior (Bartles and Zeki, 2004), close friendships (Vrtička et al, 2009), and social cooperation (Feng et al, 2015), highlighting its role in multiple human attachments and general affiliative behavior. Results indicate that both caudate and ventral straitum underpin the coparental bond, a bond built less on sexual attraction and more on long-term goals, mature partnership, and adult-adult cooperation (Aupperle et al, 2015; MendeSiedlecki et al, 2012; Zeki and Romaya, 2010).

However, within the striatum only the caudate differentiated distinct corticostriatal connectivity patterns corresponding with specific coparental styles and social neuropeptides; caudate-vmPFC connectivity increased along the dimension of coparental collaboration, while caudatedACC connectivity along the dimension of competition/ undermining. The motivational dopaminergic circuit recruits the dACC to increase vigilance to avoid contacts labeled as negative for self or child in insecure social contexts. Although aversive social processing is adaptive under certain conditions (Bromberg-Martin et al, 2010), high vigilance and lack of coparental trust may index insufficient behavior regulation that may hamper child adjustment. The dACC, a key structure of the emotional reactivity system (Etkin et al, 2015), is implicated in unpleasant experiences, stereotyping, social exclusion, detecting cognitive and emotional conflicts, threat processing, and social rejection, and is part of the rage circuitry in rodents, along with PAG, insula, and amygdala (Cacioppo et al, 2013; Eisenberger et al, 2003; Kubota et al, 2012). In contrast, in secure collaborative coparenting the dopaminergic circuit connects with the vmPFC to promote mentalization, familiarity, and cooperation. The change in caudate-vmPFC connectivity as collaborative coparenting increased complements studies on the role of vmPFC in social cognition, emotion regulation, affective foresight, and mood regulation (Abraham et al, 2014; Benoit et al, 2014; Etkin et al, 2015; Lindquist et al, 2015). Greater activity of the vmPFC has been associated with reduced activity of dACC during both stress and attachment paradigms (Eisenberger et al, 2011; Wager et al, 2009). Moreover, higher vmPFC activity is linked with reduced threat responding (Wager et al, 2009), personal closeness, prosocial behavior, longer relationship duration, and perception of relationships as supportive (Declerck et al, 2013; Eisenberger et al, 2011), attributes which mark the emotionally-stable coparental bond. Thus, findings highlight the fine-tuned balance between connectivity of the subcortical motivational circuit with either the vmPFC or dACC as a neural marker for the nature of the alliance among childrearing adults.

Findings from the whole-brain functional connectivity analyses revealed stronger corticostriatal connectivity between the caudate and $\mathrm{dlPFC}$ and $\mathrm{OOFC}$ as collaborative coparenting increased. This accords with prior research showing that both the dlPFC and IOFC are implicated in selfrelated information, a critical ability for maintaining longlasting bonds (Meshi et al, 2016). The dlPFC is involved in conflict resolution between selfish and prosocial goals (Frith, 2000; Lieberman, 2010) and both areas participate in response inhibition by switching one's behavior when socially unacceptable (Elliott et al, 2000). Such abilities are important for sharing parental practices, accepting criticism, and altering behavior according to long-term joint parenting goals, dimensions of the collaborative coparental bond.

In addition to brain-behavior links, we found that functional connectivity patterns were distinctly associated with OT and AVP; caudate-vmPFC connectivity with OT and caudate-dACC connectivity with AVP, and the dyadic path model indicated that links between corticostriatal connectivity and behavior were uniquely mediated by OT and AVP. Prior research has pinpointed the role of OT and AVP in supporting the transition to parenthood (Gordon et al, 2010), parent-infant synchrony (Apter-Levi et al, 2014), and parent's neural response to infant cues (Rilling and Young, 2014). Yet, OT and AVP support distinct configurations of social behaviors (Apter-Levi et al, 2014), and although OT increases social affiliation and buffers social stress (Feldman, 2015), AVP is implicated in aggression, antisocial behavior, and guarding relationship exclusivity (Meyer-Lindenberg et al, 2011; Neumann and Landgraf, 2012). Although both are central for social life, there is specificity in their role for bonding. Our findings show that greater OT is associated with coparental support which promotes child adaptation, whereas greater AVP indexes vigilance and competition, which may hamper child's socioemotional growth (Feldman and Masalha, 2010).

Of special interest is the finding that the parent's vmPFCcaudate connectivity was associated with lower externalizing problems as mediated by collaborative coparenting. This accords with a meta-analysis showing that collaborative coparenting is related to fewer externalizing behavior problems (Teubert and Pinquart, 2010), and with extant research indicating that children's externalizing symptoms correlate with deficits in emotion regulation (Mullin and Hinshaw, 2007). The vmPFC, which coactivated with striatum to support collaborative coparenting, plays a central role in both inter- and intrapersonal emotion regulation (Etkin et al, 2015; Hallam et al, 2014). Taken together, our findings provide the first neurobiological evidence for the role of corticostraital pathways in shaping the coparental alliance, which supports infants' adaptation to social life.

Finally, consistent with the biobehavioral perspective on affiliation (Feldman, 2017) our findings show that subtle 
coparental cues, which are distinct from the global parentchild relationship that has been the focus of most parenting research, may carry long-lasting effects on the family atmosphere and child adjustment. Findings are thus consistent with the recent call to formulate a 'two-person neuroscience' (Hari, 2015) and focus on brain mechanisms that enable the development of brain-to-brain we mode (Gallotti and Frith, 2013) by providing one example on how mutual adaptations between caregivers may generate specific neural coupling that is uniquely linked with neuropeptides and behavioral patterns to confer evolutionary advantage on the family system and its individual members (Hasson and Frith, 2016).

Limitations of the study should be considered. Our sample included middle-class partnered co-parents and future studies are needed to generalize the findings to other populations, including divorced parents, high-risk contexts, or multi-generational caregivers. Another limitation is the inability to infer causality only relationships among variables, indicating that our findings are preliminary and used an exploratory approach at the whole-brain level complemented with a priori regions of interest and can guide future research. Another possible limitation is the fact that both OT and AVP were sampled in the periphery and not centrally, and no behavioral measures were collected during or immediately after the fMRI scans. Owing to the withinmeasure change over time, we cannot clearly evaluate change over time and while the multi-method approach provides validity by showing longitudinal stability across measurement method, it is also a clear limitation by limiting the study of change over time in exact measures. Further research is required to integrate longitudinal data including brain imaging, hormonal analysis, and careful assessment of coparental behaviors across the first years of family formation.

Family systems are flexible, diverse, and adaptive to ecological and economical conditions; yet, across human history the family had carried a similar universal role. Through long-lasting cooperation within the familywhether nuclear families, extended families, or the wider network of conspecifics-adults join their effort to increase offspring fitness, facilitate feeding and protection, and help infants adapt to social life (Geary and Flinn, 2001). The coparental bond, although receiving significantly less research compared to the marital relationship, has been central to infant survival and thriving since early humanity (Hrdy, 1999). With the current social changes that redefine family bonds and the establishment of new social norms where biological parenting, romantic love, marriage, and cohabitation no longer necessarily overlap, the coparental bond is becoming a critical factor in infant development and assessing its components is of increasing importance. Our innovative findings raise the possibility that the evolutionary-ancient coparental bond, underpinned by corticostriatal pathways, may have provided a platform for the complex and unique architecture of the human social brain that supports the ability to form cooperative social affiliations. Further insight into the coparental bond and its unique expressions across a variety of family types and cultural contexts is required to elucidate the neurobiological mechanisms by which adults collaborate to facilitate the growth and socialization of human infants.

\section{FUNDING AND DISCLOSURE}

This research was supported by the German-Israeli Foundation and the Simss-Mann Foundation. The authors declare no conflict of interest.

\section{REFERENCES}

Abraham E, Hendler T, Shapira-Lichter I, Kanat-Maymon Y, Zagoory-Sharon O, Feldman R (2014). Father's brain is sensitive to childcare experiences. Proc Natl Acad Sci 111: 9792-9797.

Abraham E, Hendler T, Zagoory-Sharon O, Feldman R (2016). Network integrity of the parental brain in infancy supports the development of children's social competencies. Soc Cogn Affect Neurosci 11: 1707-1718.

Acevedo BP, Aron A, Fisher HE, Brown LL (2011). Neural correlates of long-term intense romantic love. Soc Cogn Affect Neurosci 7: 145-159.

Achenbach TM (1991). Manual for the Child Behavior Checklist/418 and 1991 Profile. Department of Psychiatry, University of Vermont: Burlington, VT, USA, p 288.

Altenburger LE, Lang SN, Schoppe-Sullivan SJ, Dush CMK, Johnson S (2015). Toddlers' differential susceptibility to the effects of coparenting on social-emotional adjustment. Int $J$ Behav Dev 41: 228-237.

Apter-Levi Y, Zagoory-Sharon O, Feldman R (2014). Oxytocin and vasopressin support distinct configurations of social synchrony. Brain Res 1580: 124-132.

Assad KK, Donnellan MB, Conger RD (2007). Optimism: an enduring resource for romantic relationships. J Pers Soc Psychol 93: 285.

Aupperle RL, Melrose AJ, Francisco A, Paulus MP, Stein MB (2015). Neural substrates of approach-avoidance conflict decision-making. Hum Brain Map 36: 449-462.

Báez-Mendoza R, Schultz W (2013). The role of the striatum in social behavior. Front Neurosci 7: 233.

Bartels A, Zeki S (2004). The neural correlates of maternal and romantic love. NeuroImage 21: 1155-1166.

Bault N, Joffily M, Rustichini A, Coricelli G (2011). Medial prefrontal cortex and striatum mediate the influence of social comparison on the decision process. Proc Natl Acad Sci 108: 16044-16049.

Beck AT (1978). Beck Depression Inventory. Harcourt Brace Jovanovich. Psychological Corporation: San Antonio, TX, USA.

Benningfield MM, Blackford JU, Ellsworth ME, Samanez-Larkin GR, Martin PR, Cowan RL et al (2014). Caudate responses to reward anticipation associated with delay discounting behavior in healthy youth. Dev Cogn Neurosci 7: 43-52.

Benoit RG, Szpunar KK, Schacter DL (2014). Ventromedial prefrontal cortex supports affective future simulation by integrating distributed knowledge. Proc Natl Acad Sci 111: 16550-16555.

Bromberg-Martin ES, Matsumoto M, Hikosaka O (2010). Dopamine in motivational control: rewarding, aversive, and alerting. Neuron 68: 815-834.

Cacioppo S, Frum C, Asp E, Weiss RM, Lewis JW, Cacioppo JT (2013). A quantitative meta-analysis of functional imaging studies of social rejection. Sci Rep 3: 2027.

Caldera YM, Lindsey EW (2006). Coparenting, mother-infant interaction, and infant-parent attachment relationships in two-parent families. J Fam Psychol 20: 275-283.

Calzavara R, Mailly P, Haber SN (2007). Relationship between the corticostriatal terminals from areas 9 and 46, and those from area $8 \mathrm{~A}$, dorsal and rostral premotor cortex and area 24c: an anatomical substrate for cognition to action. Eur J Neurosci 26: 2005-2024.

De Wit S, Watson P, Harsay HA, Cohen MX, van de Vijver I, Ridderinkhof KR (2012). Corticostriatal connectivity underlies 
individual differences in the balance between habitual and goaldirected action control. J Neurosci 32: 12066-12075.

Declerck CH, Boone C, Emonds G (2013). When do people cooperate? The neuroeconomics of prosocial decision making. Brain Cogn 81: 95-117.

Delgado MR (2007). Reward-related responses in the human striatum. Ann N Y Acad Sci 1104: 70-88.

Ditzen B, Schaer M, Gabriel B, Bodenmann G, Ehlert U, Heinrichs M (2009). Intranasal oxytocin increases positive communication and reduces cortisol levels during couple conflict. Biol Psychiatry 65: $728-731$.

Dyer J, Fagan J, Kaufman R, Pearson J, Cabrera NJ (2015). Fatherhood research and practice network coparenting relationship scale. Retrieved from http://www.frpn.org/asset/frpn-researchmeasure-coparenting-relationship-scale.

Eisenberger NI, Master SL, Inagaki TK, Taylor SE, Shirinyan D, Lieberman MD et al (2011). Attachment figures activate a safety signal-related neural region and reduce pain experience. Proc Natl Acad Sci 108: 11721-11726.

Eisenberger NI, Lieberman MD, Williams KD (2003). Does rejection hurt? An fMRI study of social exclusion. Science 302: 290-292.

Elliott R, Dolan RJ, Frith CD (2000). Dissociable functions in the medial and lateral orbitofrontal cortex: evidence from human neuroimaging studies. Cereb Cortex 10: 308-317.

Etkin A, Büchel C, Gross JJ (2015). The neural bases of emotion regulation. Nat Rev Neurosci 16: 693-700.

Farr RH, Patterson CJ (2013). Coparenting among lesbian, gay, and heterosexual couples: associations with adopted children's outcomes. Child Dev 84: 1226-1240.

Feinberg ME, Brown LD, Kan ML (2012). A multi-domain self-report measure of coparenting. Parenting 12: 1-21.

Feinberg ME (2003). The internal structure and ecological context of coparenting: a framework for research and intervention. Parenting 3: 95-131.

Feldman R (1998). Coding Interactive Behavior Manual. Bar-Ilan Univ Press: Tel Aviv, Israel.

Feldman R (2007). Parent-infant synchrony and the construction of shared timing; physiological precursors, developmental outcomes, and risk conditions. J Child Psychol Psychiatry 48: 329-354.

Feldman R (2012). Parenting behavior as the environment where children grow. Mayes LC, Lewis M (eds). The Cambridge Handbook of Environment in Human Development. Cambridge Univ Press: New York, NY, USA, pp 535-567.

Feldman R (2015). The adaptive human parental brain: implications for children's social development. Trends Neurosci 38: 387-399.

Feldman R (2016). The neurobiology of mammalian parenting and the biosocial context of human caregiving. Horm Behav 77: 3-17.

Feldman R (2017). The neurobiology of human attachments. Trends Cogn Sci 21: 80-99.

Feldman R, Masalha S (2010). Parent-child and triadic antecedents of children's social competence: cultural specificity, shared process. Dev Psychol 46: 455.

Feldman R, Masalha S, Derdikman-Eiron R (2010). Conflict resolution in the parent-child, marital, and peer contexts and children's aggression in the peer group: a process-oriented cultural perspective. Dev Psychol 46: 310.

Feldman R, Rosenthal Z, Eidelman AI (2014). Maternal-preterm skin-to-skin contact enhances child physiologic organization and cognitive control across the first 10 years of life. Biol Psychiatry 75: $56-64$.

Feng C, Lori A, Waldman ID, Binder EB, Haroon E, Rilling JK (2015). A common oxytocin receptor gene (OXTR) polymorphism modulates intranasal oxytocin effects on the neural response to social cooperation in humans. Genes Brain Behav 14: 516-525.

Frith CD (2000). The role of dorsolateral prefrontal cortex in the selection of action as revealed by functional imaging. In: Monsell
S, Driver J (eds). Control of Cognitive Processes, 18th edn. MIT Press: Cambridge, MA, USA, pp 549-565.

Gable S, Belsky J, Crnic K (1995). Coparenting during the child's 2nd year: a descriptive account. J Marriage Fam 57: 609-616.

Gallotti M, Frith CD (2013). Social cognition in the we-mode. Trends Cogn Sci 17: 160-165.

Geary DC, Flinn MV (2001). Evolution of human parental behavior and the human family. Parenting 1: 5-61.

Gettler LT (2014). Applying socioendocrinology to evolutionary models: fatherhood and physiology. Evol Anthropol Issues News Rev 23: 146-160.

Gilam G, Lin T, Raz G, Azrielant S, Fruchter E, Ariely D et al (2015). Neural substrates underlying the tendency to accept anger-infused ultimatum offers during dynamic social interactions. NeuroImage 120: 400-411.

Gilbert SF, Bosch TCG, Ledón-Rettig C (2015). Eco-evo-devo: developmental symbiosis and developmental plasticity as evolutionary agents. Nat Rev Genet 16: 611-622.

Goebel R, Esposito F, Formisano E (2006). Analysis of functional image analysis contest (FIAC) data with brainvoyager QX: from single-subject to cortically aligned group general linear model analysis and self-organizing group independent component analysis. Hum Brain Map 27: 392-401.

Gordon I, Zagoory-Sharon O, Leckman JF, Feldman R (2010). Oxytocin, cortisol, and triadic family interactions. Physiol Behav 101: 679-684.

Grahn JA, Parkinson JA, Owen AM (2008). The cognitive functions of the caudate nucleus. Prog Neurobiol 86: 141-155.

Griffin D, Gonzalez R (1995). Correlational analysis of dyad-level data in the exchangeable case. Psychol Bull 118: 430.

Haber SN, Knutson B (2009). The reward circuit: linking primate anatomy and human imaging. Neuropsychopharmacology 35: 4-26.

Hallam GP, Webb TL, Sheeran P, Miles E, Niven K, Wilkinson ID et al (2014). The neural correlates of regulating another person's emotions: an exploratory fMRI study. Front Hum Neurosci 8: 376.

Hari R (2015). Centrality of social interaction in human brain function. Neuron 88: 181-193.

Hasson U, Frith CD (2016). Mirroring and beyond: coupled dynamics as a generalized framework for modelling social interactions. Phil Trans R Soc B 371 (doi:10.1098/rstb.2015.0366).

Hayes AF (2013). Introduction to Mediation, Moderation, and Conditional Process Analysis: A Regression-Based Approach. Guilford Press: New York, NY, USA.

Hrdy SB (1999). Mother Nature: A History of Mothers, Infants, and Natural Selection. Pantheon Books: New York, NY, USA.

Hrdy SB (2011). Mothers and Others: The Evolutionary Origins of Mutual Understanding. Harvard University Press: Cambridge.

Keller J, Young CB, Kelley E, Prater K, Levitin DJ, Menon V (2013). Trait anhedonia is associated with reduced reactivity and connectivity of mesolimbic and paralimbic reward pathways. J Psychiatr Res 47: 1319-1328.

Kenny DA, Kashy DA, Cook WL (2006). The Analysis of Dyadic Data. Guilford: New York, NY, USA.

Kline RB (2005). Principles and Practice of Structural Equation Modeling (Methodology in the Social Sciences), 2nd edn. Guilford: New York, NY, USA.

Kosfeld M, Heinrichs M, Zak PJ, Fischbacher U, Fehr E (2005). Oxytocin increases trust in humans. Nature 435: 673-676.

Kubota JT, Banaji MR, Phelps EA (2012). The neuroscience of race. Nat Neurosci 15: 940-948.

Lebowitz ER, Silverman WK, Martino AM, Zagoory-Sharon O, Feldman R, Leckman JF (2017). Oxytocin response to youth-mother interactions in clinically anxious youth is associated with separation anxiety and dyadic behavior. Depress Anxiety 34: 127-136.

LeRoy M, Mahoney A, Pargament KI, DeMaris A (2013). Longitudinal links between early coparenting and infant behaviour problems. Early Child Dev Care 183: 360-377. 
Lieberman MD (2010). Social Cognitive Neuroscience. Handbook of Social Psychology. Wiley: New York, NY, USA.

Lindquist KA, Satpute AB, Wager TD, Weber J, Barrett LF (2015). The brain basis of positive and negative affect: evidence from a meta-analysis of the human neuroimaging literature. Cereb Cortex 26: 1910-1922.

McHale JP, Rasmussen JL (1998). Coparental and family grouplevel dynamics during infancy: early family precursors of child and family functioning during preschool. Dev Psychopathol 10: 39-59.

McHale JP, Irase K (2011). Coparenting in diverse family systems. In: McHale JP, Lindahl KM (eds). Coparenting-A Conceptual and Clinical Examination of Family Systems. American Psychological Association Press, Washington, DC, USA, pp 15-38.

Mechias ML, Etkin A, Kalisch R (2010). A meta-analysis of instructed fear studies: implications for conscious appraisal of threat. Neuroimage 49: 1760-1768.

Mende-Siedlecki P, Said CP, Todorov A (2012). The social evaluation of faces: a meta-analysis of functional neuroimaging studies. Soc Cogn Affect Neurosci 8: 285-299.

Meshi D, Mamerow L, Kirilina E, Morawetz C, Margulies DS, Heekeren HR (2016). Sharing self-related information is associated with intrinsic functional connectivity of cortical midline brain regions. Sci Rep 6: 22491.

Meyer-Lindenberg A, Domes G, Kirsch P, Heinrichs M (2011). Oxytocin and vasopressin in the human brain: social neuropeptides for translational medicine. Nat Rev Neurosci 12: 524-538.

Milad MR, Rauch SL (2012). Obsessive-compulsive disorder: beyond segregated cortico-striatal pathways. Trends Cogn Sci 16: 43-51.

Mobbs D, Yu R, Rowe JB, Eich H, FeldmanHall O, Dalgleish T (2010). Neural activity associated with monitoring the oscillating threat value of a tarantula. Proc Natl Acad Sci 107: 20582-20586.

Mullin BC, Hinshaw SP. Emotion regulation and externalizing disorders in children and adolescents. Gross JJ (ed). Handbook of Emotion Regulation. Guilford Press: New York, NY, USA, 2007, 523-541.

Neumann ID, Landgraf R (2012). Balance of brain oxytocin and vasopressin: implications for anxiety, depression, and social behaviors. Trends Neurosci 35: 649-659.

Numan M, Young LJ (2016). Neural mechanisms of mother-infant bonding and pair bonding: similarities, differences, and broader implications. Horm Behav 77: 98-112.

O'Doherty J, Dayan P, Schultz J, Deichmann R, Friston K, Dolan RJ (2004). Dissociable roles of ventral and dorsal striatum in instrumental conditioning Science 304: 452-454.

Olsen JA, Kenny DA (2006). Structural equation modeling with interchangeable dyads. Psychol Methods 11: 127.

Pauli WM, O’Reilly RC, Yarkoni T, Wager TD (2016). Regional specialization within the human striatum for diverse psychological functions. Proc Natl Acad Sci 113: 1907-1912.

Preacher KJ, Hayes AF (2008). Asymptotic and resampling strategies for assessing and comparing indirect effects in multiple mediator models. Behav Res Methods 40: 879-891.

Rainville P (2002). Brain mechanisms of pain affect and pain modulation. Curr Opin Neurobiol 12: 195-204.

Rilling JK, Gutman DA, Zeh TR, Pagnoni G, Berns GS, Kilts CD (2002). A neural basis for social cooperation. Neuron 35: 395-405.

Rilling JK, Sanfey AG, Aronson JA, Nystrom LE, Cohen JD (2004). Opposing BOLD responses to reciprocated and unreciprocated altruism in putative reward pathways. Neuroreport 15: 2539-2243.

Rilling JK, Young LJ (2014). The biology of mammalian parenting and its effect on offspring social development. Science 345: $771-776$.
Robinson JL, Laird AR, Glahn DC, Blangero J, Sanghera MK, Pessoa L et al (2012). The functional connectivity of the human caudate: an application of meta-analytic connectivity modeling with behavioral filtering. Neuroimage 60: 117-129.

Sabatinelli D, McTeague LM, Dhamala M, Frank DW, Wanger TJ, Adhikari BM (2015). Reduced medial prefrontal-subcortical connectivity in dysphoria: Granger causality analyses of rapid functional magnetic resonance imaging. Brain Connect 5: $1-9$.

Schneider BH, Attili G, Nadel J, Weissberg RP(eds) (1989). Social Competence in Developmental Perspective. Kluwer: Dordrecht.

Schneiderman I, Kanat-Maymon Y, Ebstein RP, Feldman R (2014). Cumulative risk on the oxytocin receptor gene (OXTR) underpins empathic communication difficulties at the first stages of romantic love. Soc Cogn Affect Neurosci 9: 1524-1529.

Schneiderman I, Zagoory-Sharon O, Leckman JF, Feldman R (2012). Oxytocin during the initial stages of romantic attachment: relations to couples' interactive reciprocity. Psychoneuroendocrinology 37: 1277-1285.

Schultz W (2000). Multiple reward signals in the brain. Nat Rev Neurosci 1: 199-207.

Silverstein LB, Auerbach CF (1999). Deconstructing the essential father. Am Psychol 54: 397.

Somerville LH, Heatherton TF, Kelley WM (2006). Anterior cingulate cortex responds differentially to expectancy violation and social rejection. Nat Neurosci 9: 1007-1008.

Spielberger CDGR, Lushene SHRE (1970). Manual for the StateTrait Anxiety Inventory. Palo Alto Consulting Psychologist Press: Palo Alto, CA, USA.

Takahashi H, Kato M, Matsuura M, Mobbs D, Suhara T, Okubo Y (2009). When your gain is my pain and your pain is my gain: neural correlates of envy and schadenfreude. Science 323: 937-939.

Teubert D, Pinquart M (2010). The association between coparenting and child adjustment: a meta-analysis. Parent Sci Pract 10: 286-307.

Tops M, Koole SL, IJzerman H, Buisman-Pijlman FT (2014). Why social attachment and oxytocin protect against addiction and stress: insights from the dynamics between ventral and dorsal corticostriatal systems. Pharmacol Biochem Behav 119: $39-48$.

US Census Bureau (2014). American community survey. Retrieved from https://www.census.gov/programs-surveys/acs/news/datareleases/2014/release.html.

Vakart A, Apter-Levi Y, Feldman R (2017). Fathering moderates the effects of maternal depression on the family process. Dev Psychopathol (e-pub ahead of print).

Van den Bos R (2015). The dorsal striatum and ventral striatum play different roles in the programming of social behaviour: a tribute to Lex Cools. Behav Pharmacol 26: 6-17.

Vrtička P, Andersson F, Sander D, Vuilleumier P (2009). Memory for friends or foes: the social context of past encounters with faces modulates their subsequent neural traces in the brain. Soc Neurosci 4: 384-401.

Vrtička P, Sander D, Vuilleumier P (2011). Effects of emotion regulation strategy on brain responses to the valence and social content of visual scenes. Neuropsychologia 49: 1067-1082.

Wager TD, Barrett LF, Bliss-Moreau E, Lindquist K, Duncan S, Kober $\mathrm{H}$ et al (2008). The neuroimaging of emotion. In: Lewis M, et al. (eds). The Handbook of Emotions, 3rd edn. Guilford: New York, NY, USA, pp 249-271.

Wager TD, Waugh CE, Lindquist M, Noll DC, Fredrickson BL, Taylor SF (2009). Brain mediators of cardiovascular responses to social threat. Part I: reciprocal dorsal and ventral sub-regions of the medial prefrontal cortex and heart-rate reactivity. Neuroimage 47: $821-835$. 
Weisman O, Schneiderman I, Zagoory-Sharon O, Feldman R (2013). Salivary vasopressin increases following intranasal oxytocin administration. Peptides 40: 99-103.

Wilson EO (2014). The Meaning of Human Existence. WW Norton \& Company: New York, NY, USA.

Woo CW, Krishnan A, Wager TD (2014). Cluster-extent based thresholding in fMRI analyses: pitfalls and recommendations. Neuroimage 91: 412-419.
Woody E, Sadler P (2005). Structural equation models for interchangeable dyads: being the same makes a difference. Psychol Methods 10: 139.

Zeki S, Romaya JP (2010). The brain reaction to viewing faces of opposite- and same-sex romantic partners. PLoS ONE 5: e15802.

Zink CF, Tong Y, Chen Q, Bassett DS, Stein JL, Meyer-Lindenberg A (2008). Know your place: neural processing of social hierarchy in humans. Neuron 58: 273-283.

Supplementary Information accompanies the paper on the Neuropsychopharmacology website (http://www.nature.com/npp) 